\title{
Apparent Barrier Height in Scanning Tunneling Microscopy Revisited
}

Olesen, L.; Brandbyge, Mads; Sørensen, Mads Reinholdt; Jacobsen, Karsten Wedel; Lægsgaard, E.; Stensgaard, I.; Besenbacher, Flemming

Published in:

Physical Review Letters

Link to article, DOI:

10.1103/PhysRevLett.76.1485

Publication date:

1996

Document Version

Publisher's PDF, also known as Version of record

Link back to DTU Orbit

Citation (APA):

Olesen, L., Brandbyge, M., Sørensen, M. R., Jacobsen, K. W., Lægsgaard, E., Stensgaard, I., \& Besenbacher, F. (1996). Apparent Barrier Height in Scanning Tunneling Microscopy Revisited. Physical Review Letters, 76(9), 1485-1488. https://doi.org/10.1103/PhysRevLett.76.1485

\section{General rights}

Copyright and moral rights for the publications made accessible in the public portal are retained by the authors and/or other copyright owners and it is a condition of accessing publications that users recognise and abide by the legal requirements associated with these rights.

- Users may download and print one copy of any publication from the public portal for the purpose of private study or research.

- You may not further distribute the material or use it for any profit-making activity or commercial gain

- You may freely distribute the URL identifying the publication in the public portal 


\title{
Apparent Barrier Height in Scanning Tunneling Microscopy Revisited
}

\author{
L. Olesen, ${ }^{1}$ M. Brandbyge, ${ }^{2}$ M. R. Sørensen, ${ }^{2}$ K. W. Jacobsen, ${ }^{2}$ E. Lægsgaard, ${ }^{1}$ I. Stensgaard, ${ }^{1}$ F. Besenbacher ${ }^{1}$ \\ ${ }^{1}$ Center for Atomic-scale Materials Physics (CAMP), Institute of Physics and Astronomy, University of Aarhus, \\ DK 8000 Aarhus C, Denmark \\ ${ }^{2}$ Center for Atomic-scale Materials Physics (CAMP), Physics Department, Technical University of Denmark, \\ DK 2800 Lyngby, Denmark
}

(Received 3 November 1995)

\begin{abstract}
The apparent barrier height $\phi_{\text {ap }}$, that is, the rate of change of the logarithm of the conductance with tip-sample separation in a scanning tunneling microscope (STM), has been measured for $\mathrm{Ni}$, Pt, and $\mathrm{Au}$ single crystal surfaces. The results show that $\phi_{\text {ap }}$ is constant until point contact is reached rather than decreasing at small tunneling gap distances, as previously reported. The findings for $\phi_{\text {ap }}$ can be accounted for theoretically by including the relaxations of the tip-surface junction in an STM due to the strong adhesive forces at close proximity. These relaxation effects are shown also to be generally relevant under imaging conditions at metal surfaces.
\end{abstract}

PACS numbers: 61.16.Ch

Although the scanning tunneling microscope (STM) by now is a well established and powerful instrument for the atomic scale imaging of clean and adsorbate covered surfaces, detailed information on the tunneling barrier and in particular on the barrier height is still very scarce. As a consequence of this, the existing STM theories are still not able to account quantitatively for the experimental STM images, e.g., the measured corrugation heights and their variation with tip-surface distance, even for a clean, unreconstructed metal surface. It has been pointed out ever since the pioneering study by Binnig, Roher, and coworkers [1] that information on the tunneling barrier could be used to give information on, e.g., the chemical specificity of the surface, the local electronic charge, and band-bending effects on semiconductor surfaces. A number of different ways to extract information on the tunneling barrier have been presented. In a simple onedimensional tunneling model, the tunneling conductance $G_{t}$ in STM can be written as

$$
G_{t}=I_{t} / V_{t} \propto \exp (-1.025 \sqrt{\bar{\phi}} z) \quad(\mathrm{eV} \text { and } \AA),
$$

where $I_{t}$ and $V_{t}$ are the tunneling current and voltage, respectively, $\bar{\phi}$ is the average barrier height, and $z$ is the tip-surface distance. Motivated by this equation, it has been generally accepted to define an apparent barrier height as

$$
\phi_{\mathrm{ap}}=\left(\frac{1}{1.025} \frac{d\left(\ln G_{t}\right)}{d z}\right)^{2} .
$$

It is clear from Eq. (2) that $\phi_{\text {ap }}$ can be obtained by measuring, or calculating, $G_{t}$ as a function of $z$. However, conflicting experimental conclusions regarding the behavior of $\phi_{\text {ap }}$ as a function of gap distance have been reported in the past. In most studies, it is concluded that $\phi_{\text {ap }}$ decreases for $z \leq 6 \AA[2-5]$. On the other hand, Dürig, Züger, and Pohl found, in the case of an Ir tipsample system, that $\phi_{\text {ap }}$ is constant all the way to point contact [6], a fact which was attributed to an increased participation of the $d$ electrons in Ir at small tunneling gap widths, accidentally counteracting the collapse of the barrier. It has also been reported that as the tip-surface separation decreases, $\phi_{\text {ap }}$ initially increases at a tip-surface distance of $\approx 2 \AA$ followed by a sudden decrease within a fraction of an angstrom [7].

Theoretically, a detailed analysis of the apparent barrier height has been performed by Lang [8]. He has pointed out that it is of crucial importance to realize the atomistic three-dimensional nature of the problem when tip and surface are in close proximity [8]. In this case, the potential energy surface for the electrons between tip and surface forms a narrow hole through which there is no direct barrier for tunneling. Nevertheless, an effective barrier arises because the constriction of the electronic motion in the direction perpendicular to the hole implies a minimum kinetic energy associated with motion in this direction [8-10]. Taking these effects into account, Lang calculated $I_{t}$ for a fixed $V_{t}$ within the local density approximation (LDA) and found that $\phi_{\text {ap }}$ decreases below the sample work function $\Phi$ at $z \approx 6 \AA$ and that it has dropped by a factor of 2 at $z \approx 4 \AA$.

In this Letter, we present a comprehensive study of $\phi_{\text {ap }}$ for $\mathrm{Au}, \mathrm{Ni}$, and Pt single crystal surfaces to try to clarify the situation experimentally. We find for all the metals studied that $\phi_{\text {ap }}$ does not decrease at small tunneling gap distances, as expected from Lang's calculations, but rather stays constant until a point contact between tip and surface is established. It is demonstrated that when measuring the variation of $\phi_{\text {ap }}$ with tip-surface separation, it is of utmost importance to measure the variation of both $I_{t}$ and $V_{t}$, and it is suggested that the neglect of the variation in $V_{t}$ may be the cause of the previously conflicting experimental results for $\phi_{\mathrm{ap}}$. Furthermore, we present a theoretical model, which shows that the decrease in $\phi_{\text {ap }}$, as predicted by Lang for a static STM geometry, is counterbalanced by contributions due to adhesive forces between tip and surface. This results in a $\phi_{\text {ap }}$ which is nearly constant as 
a function of tip-surface distance in accordance with the experimental findings.

The experiments were performed on single crystal $\mathrm{Ni}(100), \operatorname{Pt}(100)$, and $\mathrm{Au}(110)$ surfaces with a compact, high stability UHV STM [11]. Prior to an experiment, the clean surface is scanned in a constant-current mode, and atomic resolution is obtained with typical tunneling parameters $I_{t} \approx 5 \mathrm{nA}$ and $V_{t} \approx 5 \mathrm{mV}$, which correspond to a tunnel resistance of the order of $1 \mathrm{M} \Omega$, or equivalently, a conductance of the order of $1 \mu \mathrm{S}(1$ siemens $=$ $\left.1 \Omega^{-1}\right)$. At a given preselected location in the usual $x-y$ scan, the feedback loop is opened and the indentation is performed, that is, the tip is retracted $\approx 15 \AA$ to a position where it is allowed to relax for $\approx 1 \mathrm{~ms}$ before it is driven towards the surface at a rate of $\approx 500 \AA / \mathrm{s}$. During this approach of the tip $I_{t}, V_{t}$, and thus $G_{t}$ are measured at 256 points (Fig. 1).

In this connection, it is important to realize that the actual voltage across the junction $\left(V_{t}\right)$ is not at all a constant equal to the applied bias voltage $\left(V_{\text {bias }}\right)$, but rather decreases significantly as the tip-surface distance decreases [Fig. 1(a)]. This is due to the finite input impedance of the

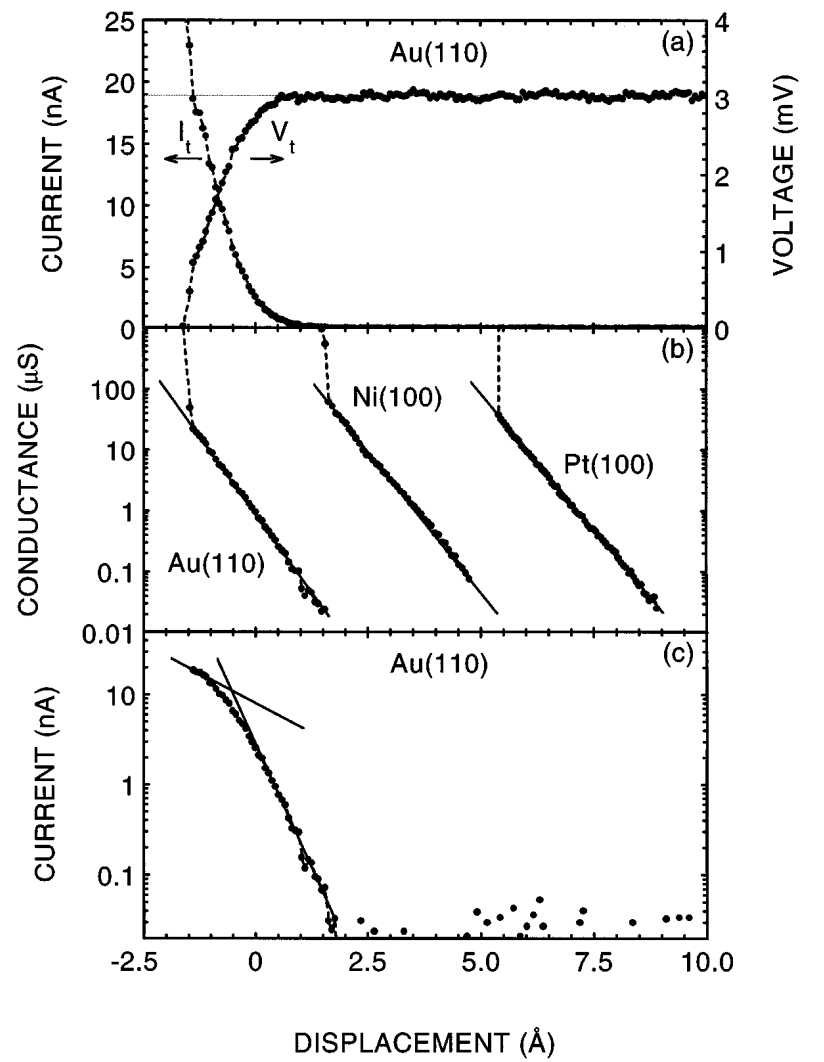

FIG. 1. $I_{t}, V_{t}$ (a), and corresponding conductance $G_{t}$ (b) measured during tip approach on $\mathrm{Au}(110)$. The resulting curves for $\mathrm{Ni}(100)$ and $\mathrm{Pt}(100)$ are also shown in (b) (displaced horizontally 3 and $7 \AA$, respectively). For all three metals, the conductance increases exponentially giving rise to a constant $\phi_{\text {ap }}$. If $\phi_{\text {ap }}$ was deduced solely from $I_{t}$ assuming a constant $V_{t}$ then one would reach the conclusion that $\phi_{\text {ap }}$ decreases from 5.1 to $0.3 \mathrm{eV}$ during tip approach (c). current preamplifier (in the present case $116 k \Omega$ ) since the applied bias voltage $V_{\text {bias }}$ is divided between this input impedance and the STM junction resistance, which can be as low as $\sim 100 \Omega$ at close proximity $[12,13]$. We circumvent this problem by introducing a low noise $(\sim$ $\left.3 \mu V_{\mathrm{rms}}\right)$ high impedance $(>1 \mathrm{~T} \Omega)$ voltage preamplifier which measures $V_{t}$ directly across the tunnel junction.

For all three surfaces, it is found that the measured values of $G_{t}$ increase exponentially in the tunneling regime until a dramatic increase in $G_{t}$ sets in [Fig. 1(b)]. At this point, the adhesive forces become so strong that tip and surface jump into point contact [14], and during the subsequent retraction of the tip from the surface a connective neck is formed $[2,13,15,16]$. It appears that results such as those depicted in Fig. 1 are reproducible only after several indentations have been performed since, in this case, the original $\mathrm{W}$ tip is probably covered with a layer of substrate material, and thus we are effectively measuring $\phi_{\text {ap }}$ for, e.g., a $\mathrm{Au}$ tip approaching a $\mathrm{Au}$ surface. From the experimental findings, we conclude that the measured apparent barrier height $\phi_{\text {ap }}$ stays constant until point contact is established. The average values for $\phi_{\text {ap }}$ deduced from more than 200 indentations on each surface are $4.7 \pm 1.0 \mathrm{eV}$ for $\mathrm{Au}(110), 4.5 \pm$ $0.7 \mathrm{eV}$ for $\mathrm{Ni}(100)$, and $3.4 \pm 0.8 \mathrm{eV}$ on $\mathrm{Pt}(100)$. The stated uncertainties reflect variations in $\phi_{\text {ap }}$ from one indentation to another due to tip geometry effects and local variations of $\phi_{\text {ap }}$ within the surface unit cell.

It should be pointed out that we would have reached an erroneous conclusion for the $z$ dependence of $\phi_{\text {ap }}$ had we not measured $V_{t}$ directly but instead assumed that $V_{t}=V_{\text {bias }}$ during the indentation. From Fig. 1(c), it is obvious that an evaluation of $\phi_{\text {ap }}$ exclusively from variations in $I_{t}$ would result in a $\phi_{\text {ap }}$ which decreases as the gap width is diminished as actually reported in several previous studies [2-5].

In the following, we shall compare our experimental findings with the results of a conductance calculation which includes the full three-dimensional character of the electronic potential between tip and surface. This static calculation of $G_{t}$ is carried out for a geometry similar to the one used by Lang and Lang, Yacoby, and Imry $[8,17]$ with a single atom between to jellium surfaces. The tipsurface separation, however, is obtained from a simulation of the atomic structure of the junction.

The conductance calculation is carried out as follows: The electron density is calculated as a simple superposition of the density from a $\mathrm{Au}$ (tip) atom and from the two jellium surfaces with $r_{s}=3$ bohrs, appropriate for Au. The potential is then obtained from this electron density as the electrostatic potential plus the exchange-correlation term in an LDA approximation. For large tip sample separations, LDA will not describe the image potential effects well, but calculations for a jellium surface which go beyond LDA suggest that this will play only a minor role at close tip-surface proximity [18]. The conduc- 
tance is calculated from the transmission coefficient for the motion of an electron from one jellium surface to the other through the tip atom using the Landauer expression for ballistic electron transport [19]. This approach has been discussed elsewhere in relation to point source emission [17] and conductance quantization in small contacts $[9,13,20]$. The transmission coefficient is calculated using a recursive coupled-channel technique with a plane-wave basis set. The method is described in detail by Hirose and Tsukada [10,21].

In the conductance calculation, we use the actual distances from the tip atom to the jellium surfaces as calculated from atomistic simulations. The tip is modeled by a pyramid shaped structure [see inset in Fig. 2(a)] consisting of nine $\mathrm{Au}(100)$ layers with $1,4,9, \ldots$, and 81 atoms, respectively. As a substrate we use a slab of nine $\mathrm{Au}(100)$ layers with $12 \times 12$ atoms in each layer. The atoms in the three topmost layers of the tip and the three bottom layers in the substrate are static. The interactions between the atoms are described by potentials derived from the effective medium theory (EMT) [22]. These potentials, which have been used in studies of several metal surface properties including reconstructions and premelt-
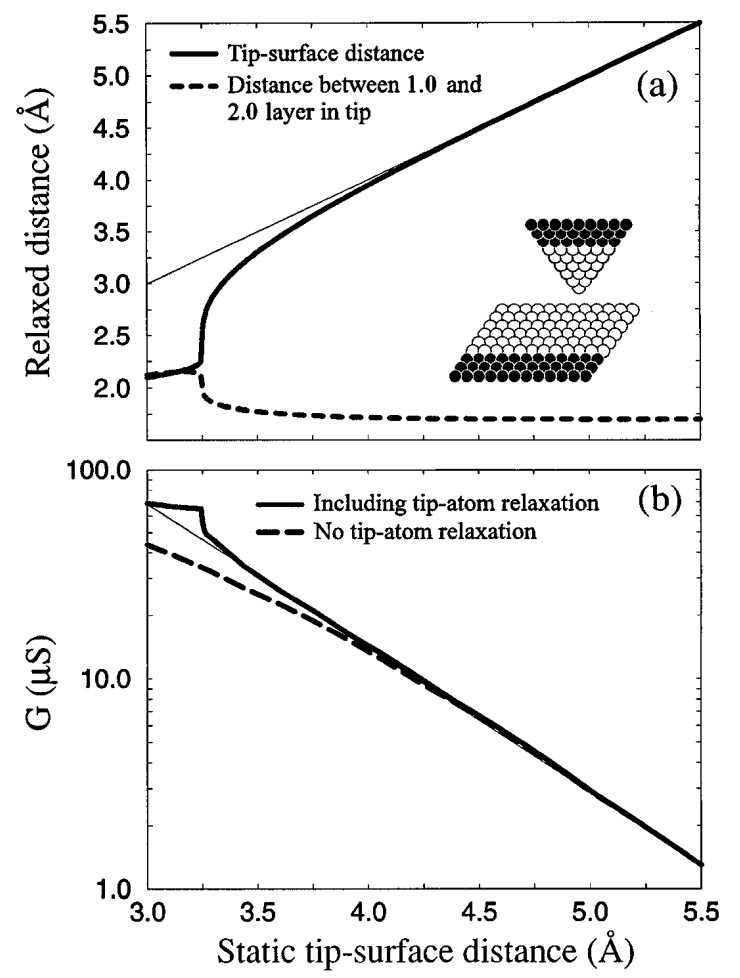

FIG. 2. (a) The actual distance between the tip atom and surface as a function of the static distance (defined as the distance between tip atom and surface in the absence of tipsurface forces). The inset shows the configuration used in the simulation. (b) The calculated conductance as a function of the static distance in the case where relaxations are taken into account (full curve) and when they are not (dashed curve). For the latter curve, the corresponding apparent barrier height varies from about $3.5 \mathrm{eV}$ at a separation of $6 \AA$ to $0.6 \mathrm{eV}$ at $3 \AA$. ing, describe small atomic displacements reasonably well. In the case of $\mathrm{Au}$ the calculated change in interatomic bond length between first and second layer atoms of the missing-row reconstructed (110) surface is, for example, $5.4 \%$ of the interlayer spacing which is quite close to the experimental value of $5.0 \%$ [23].

In the simulations, the static part of the tip is lowered towards the surface in steps of $0.005 \AA$. Between each step, the atomic positions are relaxed by a minimization procedure similar to steepest descent (see [24] for details). For comparison, we have also performed molecular dynamics simulations of the tip-surface approach at $300 \mathrm{~K}$. The results are rather similar to the ones obtained with the minimization technique, but the jump to contact appears about $0.2 \AA$ further from the surface in the finite temperature simulations because of the thermal fluctuations. We have also tried other tip geometries, e.g., a more blunt [110] oriented tip with a single apex atom. The results differ in the details, but the overall behavior is the same.

As the tip approaches the surface, the tip is stretched due to the attractive, adhesive forces acting between tip and surface. Furthermore, the atoms in the surface below the tip apex are displaced towards the tip. These relaxations imply that the actual separation between tip and surface is smaller than if the tip and surface were static. Approximately $\frac{1}{4}$ of the total shortening of the tipsurface distance is provided by an increase of the bond length between the apex atom and the four atoms in the next layer of the tip. The relaxations of the atoms in the rest of the tip contribute with approximately $\frac{1}{2}$, and the remaining $\frac{1}{4}$ arises from the displacements of the surface atoms. The largest total deformation displacement is $1.0 \AA$ immediately after the jump which occurs at an unrelaxed separation of $3.2 \AA$. The relaxations just before the jump are approximately half of those after the jump. The actual tip-surface distance is shown in Fig. 2(a) as a function of the unrelaxed tip-surface distance.

In Fig. 2(b), we show the conductance calculated in the case of a Au tip and a Au surface. The dashed curve shows how a saturation of the conductance begins to set in, in the case of a static tip atom, which corresponds to a decreasing $\phi_{\text {ap }}$ for decreasing tip-surface distance. This is equivalent to the result obtained by Lang [8]. When the relaxations are included (full curve), the saturation is compensated for by the attraction of the tip atom towards the surface over a distance of about $1 \AA$. At a closer approach, the relaxation effect even dominates so that the conductance increases slightly faster than exponentially before the jump to contact appears. This last behavior is observed in Pt break junction experiments at low temperature $(1.3 \mathrm{~K})$ [25]. The reason why this is not observed at room temperature may be that the jump to contact can occur farther from the surface. This can be caused either by thermal fluctuations, as observed in the simulations, or because activated processes which are not described on the time scale of the molecular 
dynamics simulations become possible as the temperature is raised.

In conclusion, by measuring the variations in both $I_{t}$ and $V_{t}$, we have found that the apparent barrier height $\phi_{\text {ap }}$ stays constant as a function of tip surface distance all the way to point contact. This contradicts the earlier theoretical prediction obtained with a static model of the STM junction, that $\phi_{\text {ap }}$ decreases with decreasing gap width. Since we find the same functional dependence for all the metals investigated, $\mathrm{Ni}, \mathrm{Pt}$, and $\mathrm{Au}$, we can exclude that conductance contributions from $d$ electrons can be the cause of a nondecreasing $\phi_{\text {ap }}$, as previously suggested for the case of Ir [6]. Instead we have shown that we can account for the experimental findings by including the strong attractive forces between tip and surface at small gap widths. These forces cause the actual tip-surface distance to decrease faster than dictated by the $z$-piezo element, and therefore the conductance rises faster than expected in a static model. The distance dependence of $\phi_{\text {ap }}$ is thus seen to be inevitably connected to the tip-surface interaction. However, since the tunneling parameters used in the present study cover a broad range of STM experiments, the findings are of general interest. For instance they demonstrate that a quantitative description of STM images on metal surfaces, e.g., the ability to account for the measured corrugation heights and their variation with $z$, must include the effects of tipsurface interactions [26-28]. Furthermore, it is obvious that the relaxations in the junction play a crucial role for the ability to push and drag species on metal surfaces in the field of atomic manipulation [29].

CAMP, the Center for Atomic-scale Materials Physics, is sponsored by the Danish National Research Foundation. Further funding has been obtained from the Danish Research Councils through the Center for Nano-Tribology.

[1] G. Binnig, H. Rohrer, Ch. Gerber, and E. Weibel, Appl. Phys. Lett. 40, 178 (1982).

[2] J. K. Gimzewski and R. Möller, Phys. Rev. B 36, 1284 (1987).

[3] Y. Kuk and P. J. Silverman, J. Vac. Sci. Technol. A 8, 289 (1990).

[4] R. Berndt, J. K. Gimzewski, and R. R. Schlittler, Ultramicroscopy 42-44, 528 (1992).

[5] Ph. Avouris, I.-W. Lyo, and Y. Hasegawa, J. Vac. Sci. Technol. A 11, 1725 (1993).

[6] U. Dürig, O. Züger, and D. W. Pohl, Phys. Rev. Lett. 65, 349 (1990).
[7] C. J. Chen and R. J. Hamers, J. Vac. Sci. Technol. B 9, 503 (1991).

[8] N. D. Lang, Phys. Rev. B 37, 10395 (1988); 36, 8173 (1987).

[9] E. Tekman and S. Ciraci, Phys. Rev. B 43, 7145 (1991).

[10] K. Hirose and M. Tsukada, Phys. Rev. B 51, 5278 (1995).

[11] L. Eierdal, F. Besenbacher, E. Lægsgaard, and I. Stensgaard, Surf. Sci. 312, 31 (1994).

[12] E. Lægsgaard, L. Olesen, I. Stensgaard, and F. Besenbacher (to be published).

[13] M. Brandbyge, J. Schiøtz, M. R. Sørensen, P. Stoltze, K. W. Jacobsen, J. K. Nørskov, L. Olesen, E. Lægsgaard, I. Stensgaard, and F. Besenbacher, Phys. Rev. B 52, 8499 (1995).

[14] J. B. Pethica and W. C. Oliver, Phys. Scr. T19, 61 (1987).

[15] U. Landman, W. D. Luedtke, N. A. Burnham, and R. J. Colton, Science 248, 454 (1990).

[16] L. Olesen, E. Lægsgaard, I. Stensgaard, F. Besenbacher, J. Schiøtz, P. Stoltze, K. W. Jacobsen, and J. K. Nørskov, Phys. Rev. Lett. 72, 2251 (1994); 74, 2147 (1995).

[17] N. D. Lang, A. Yacoby, and Y. Imry, Phys. Rev. Lett. 63, 1499 (1989).

[18] A. G. Eguiluz, M. Heinrichsmeier, A. Fleszar, and W. Hanke, Phys. Rev. Lett. 68, 1359 (1992).

[19] R. Landauer, J. Phys. C 1, 8099 (1989).

[20] C. W. J. Beenakker and H. van Houten, in Solid State Physics: Advances in Research and Applications, edited by H. Ehrenreich and D. Turnbull (Academic, New York, 1991), Vol. 44; J. A. Torres, J. I. Pascual, and J. J. Sáenz, Phys. Rev. B 49, 16581 (1994).

[21] We use a square supercell with a side length of 30 bohrs. The current flowing outside a square with an area corresponding to four $\mathrm{Au}$ atoms (as in the atomistic simulation) and centered at the tip atom position is subtracted. A similar approach is used by Lang [8].

[22] K. W. Jacobsen, N. K. Nørskov, and M. J. Puska, Phys. Rev. B 35, 7423 (1987); K. W. Jacobsen, Comments Cond. Matter Phys. 14, 129 (1988).

[23] W. Moritz and D. Wolf, Surf. Sci. 163, L655 (1985).

[24] M. R. Sørensen, K. W. Jacobsen, and P. Stoltze, Phys. Rev. B 53, 2101 (1996).

[25] J. M. Krans, C. J. Muller, I. K. Yanson, Th. C. M. Govaert, R. Hesper, and J.M. van Ruitenbeek, Phys. Rev. B 48, 14721 (1993).

[26] A. R. H. Clarke, J. B. Pethica, J. A. Nieminen, F. Besenbacher, E. Lægsgaard, and I. Stensgaard (to be published).

[27] G. Doyen, D. Drakova, J. V. Barth, R. Schuster, T. Gritsch, R. J. Behm, and G. Ertl, Phys. Rev. B 48, 1738 (1993).

[28] J. Wintterlin, J. Wiechers, H. Brune, T. Gritsch, H. Höfer, and R. J. Behm, Phys. Rev. Lett. 62, 59 (1989).

[29] J. A. Stroscio and D. M. Eigler, Science 254, 1319 (1991). 